\title{
apex: A new commercial off-the-shelf on- board computer platform for sounding rockets
}

Cite as: Rev. Sci. Instrum. 90, 105101 (2019); https://doi.org/10.1063/1.5118855

Submitted: 05 July 2019 . Accepted: 16 September 2019 . Published Online: 01 October 2019

N. Maas (D), R. Willnecker, R. Hemmersbach, and J. Hauslage

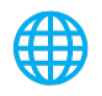

starting at $\$ 6,210$ \\ Lock-in Amplifiers up to $600 \mathrm{MHz}$}

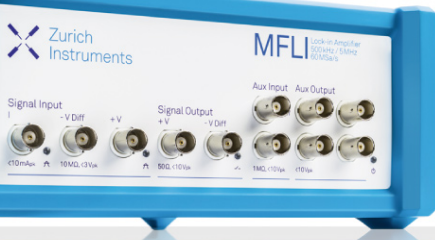

- Zurich

Instruments

Watch the Video 


\title{
apex: A new commercial off-the-shelf on-board computer platform for sounding rockets
}

\author{
Cite as: Rev. Sci. Instrum. 90, 105101 (2019); doi: 10.1063/1.5118855 \\ Submitted: 5 July 2019 - Accepted: 16 September 2019• \\ Published Online: 1 October 2019
}

\author{
N. Maas, ${ }^{1, a, b)}$ (D) Willnecker, ${ }^{1, b)}$ R. Hemmersbach, ${ }^{2}$ and J. Hauslage ${ }^{2}$
}

\begin{abstract}
AFFILIATIONS
${ }^{1}$ Microgravity User Support Center (MUSC), German Aerospace Center (DLR), 51147 Köln, Germany

${ }^{2}$ Institute of Aerospace Medicine, Gravitational Biology, German Aerospace Center (DLR), 51147 Köln, Germany
\end{abstract}

Note: This paper is part of the Special Collection: Materials and Life Science Experiments for the Sounding Rocket MAPHEUS.

a) Electronic mail: nico.maas@dlr.de.

b) URL: https://www.dlr.de/musc.

\begin{abstract}
In order to supersede the aging Microchip ATMEGA328P as the de facto standard for Commercial off-the-shelf (COTS) On-Board Computers (OBCs) with a more powerful system for different kinds of high-speed sensors and image acquisition applications, we developed advanced processors, encryption, and security experiment (apex). The platform consisting of a newly developed OBC using COTS components has been flight tested during the ATEK/MAPHEUS- 8 sounding rocket campaign. The main advantages of the apex OBC lies in the speed and simplicity of the design while maintaining operational security with a redundant master-master microcontroller system, as well as dual flash storage within each master. Additionally, a single board computer with a containerized and failure-resistant Operating System (OS) (balenaOS) was included to allow usage of a high-definition camera or other more compute-intensive tasks. The bench and flight tests were performed successfully and already showed feasible ways to further improve operational performance.
\end{abstract}

Published under license by AIP Publishing. https://doi.org/10.1063/1.5118855

\section{INTRODUCTION}

With the emergence of cost-efficient "hobbyist"/Commercial off-the-shelf (COTS) hardware like the Arduino, an easy to use and reliable microcontroller platform using the Microchip ATMEGA became available to technical enthusiasts and hobbyists in 2005 . In 2013, the first CubeSat using an ATMEGA/Arduino based OnBoard Computer (OBC), ARDUSAT, was deployed from the International Space Station (ISS) using the Nanoracks service.

Following this crowdfunded mission, different entities, started to review the effects of radiation on COTS components such as the Arduino Microcontroller Unit (MCU) and Raspberry Pi (RPi) Single Board Computer (SBC). Daniel Violettes $(\text { NASA })^{2}$ testing resulted in the Arduino failing after $56 \mathrm{krad}$ of gamma irradiation and the RPi showing problems with the universal serial bus (USB) hub at $60 \mathrm{krad}$; however, the rest of the SBC remained functional after delivering the maximum dose of $150 \mathrm{krad}$.

At the same time, the commercial partner of ARDUSAT, Spire Global, Inc., started to provide CubeSat services to corporations as well as K-12 schools, ${ }^{4}$ using the aforementioned COTS components to create extremely cost-efficient CubeSats.

However, such components were not only used in CubeSats but also in experiments of sounding rockets such as $\mathrm{MemEx}^{5}$ on board of the MAPHEUS- 6 and ATEK/MAPHEUS- 8 missions.

The biggest drawback of the ATMEGA328P MCU, which is commonly used in this application, is its age and now limited performance. Although it is still useful for simple applications, higher throughput data sampling, complex mathematics, or cryptographic functions require more computational power. To support more ambitious projects, advanced processors, encryption, and security experiment (apex) has been designed to flight-test a two-staged OBC using state-of-the-art COTS components to support different mission requirements.

\section{SYSTEM DESIGN}

As mentioned, apex consists of two different systems to support different mission roles. 
The first system is a redundant master-master configuration of two ESP32-PICO-D4 MCUs, each connected to its own 32 GB micro secure digital memory card $(\mu \mathrm{SD})$. This system is tasked with the acquisition, processing, storage, and transmission of Inter Integrated Circuit (i2c) sensor data (Figs. 1-4).

The second system is a Raspberry Pi Zero W, hardened with a specialized read-only Operating System (OS), which exposes a container hypervisor to run different mission-payloads in an isolated, managed, and noninterruptive context. It uses a $64 \mathrm{~GB} \mu \mathrm{SD}$ card. The RPi is also connected to the telemetry and signal interface of the MAPHEUS service module-as well as to the i2cbus of the first system. However, the main objective of the RPi is to record footage using a high definition (HD) camera and $\log$ occurrences of the different service module signals for later analysis (Fig. 1).

In contrast to the regularly used ATMEGA328P, the performance of the ESP32 is a massive improvement-changing from a single $20 \mathrm{MHz}, 8$ bit core to a $240 \mathrm{MHz}, 32$ bit dual core with multiple cryptographic extensions, a specialized high-speed SD card interface, as well as an "ultralow power" coprocessor for management and lightweight operations (Table I).

However, as the ESP32 has not been used in the context of space flight, this first iteration of apex was built with the purpose of testing the new MCU, while maintaining its maximum CPU clock of $240 \mathrm{MHz}$. To achieve optimal operational security, two ESP32 have been outfitted to apex, each possessing its own $\mu$ SD card and a power control circuit to be able to reset the $\mu \mathrm{SD}$ card in case of errors. While the $\mu \mathrm{SD}$ card was used to save all processed data from the $\mathrm{i} 2 \mathrm{c}$ sensors, each ESP32 had additional access to an 2.7 MB on-board SPI Flash Filesystem (SPIFFS), which was used as ring-memory to save each fiftieth data packet. In case that one complete ESP32 MCU would be rendered in-operable and the second one develop a damaged $\mu \mathrm{SD}$ card, it would hence still be able to recover data from the SPIFFS of the second ESP32.

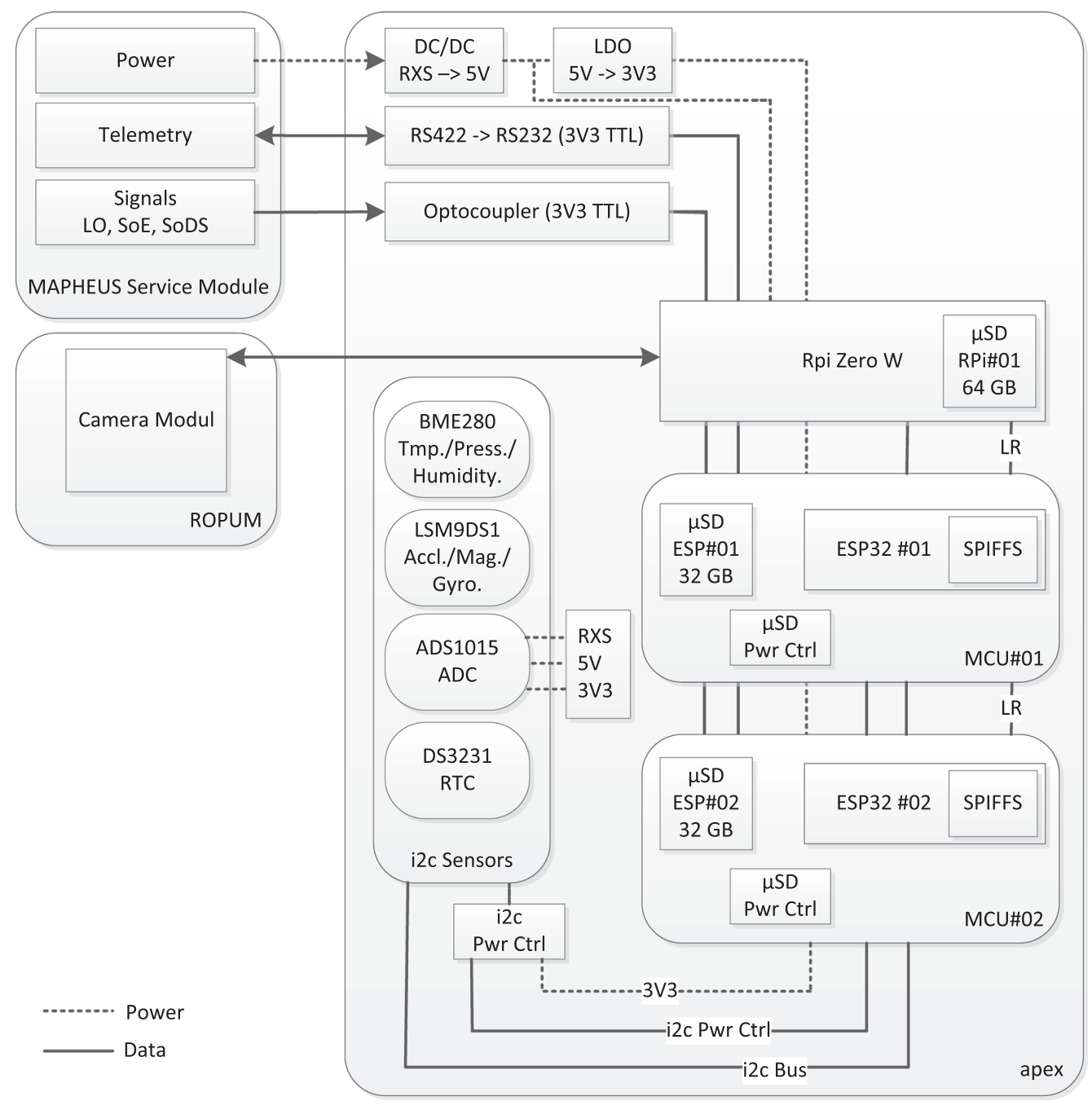

FIG. 1. apex system architecture. 


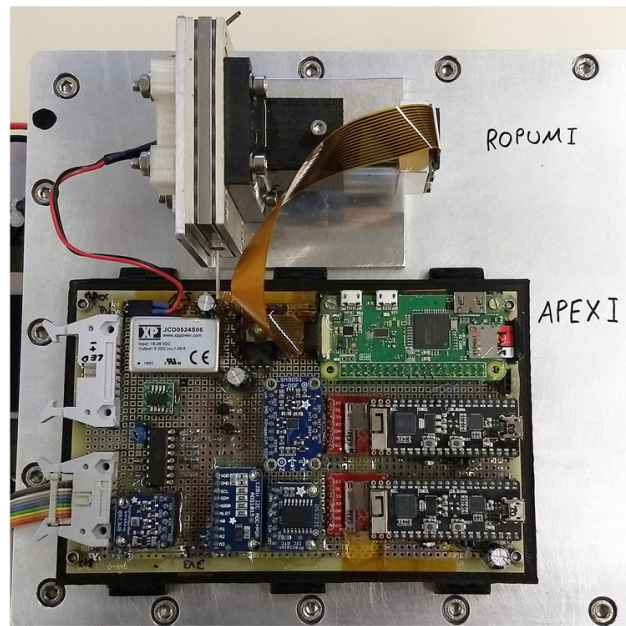

FIG. 2. apex and Rotation of Paramecium Under Microgravity (ROPUM) mounted on Cellfix II.

Both MCUs have been used in a redundant master-master configuration, accessing the i2c-bus mutually one after the other. To coordinate the utilization of the sensors, a Link Ready (LR) system has been developed. This Carrier Sense Multiple Access/Collision Avoidance (CSMA/CA) ${ }^{6}$ inspired system uses an additional connection, spanning both MCUs as well as the RPi. Whenever one of the three systems wishes to access the sensors, it will check the status of the LR-whether it has been pulled to a low logic level, meaning

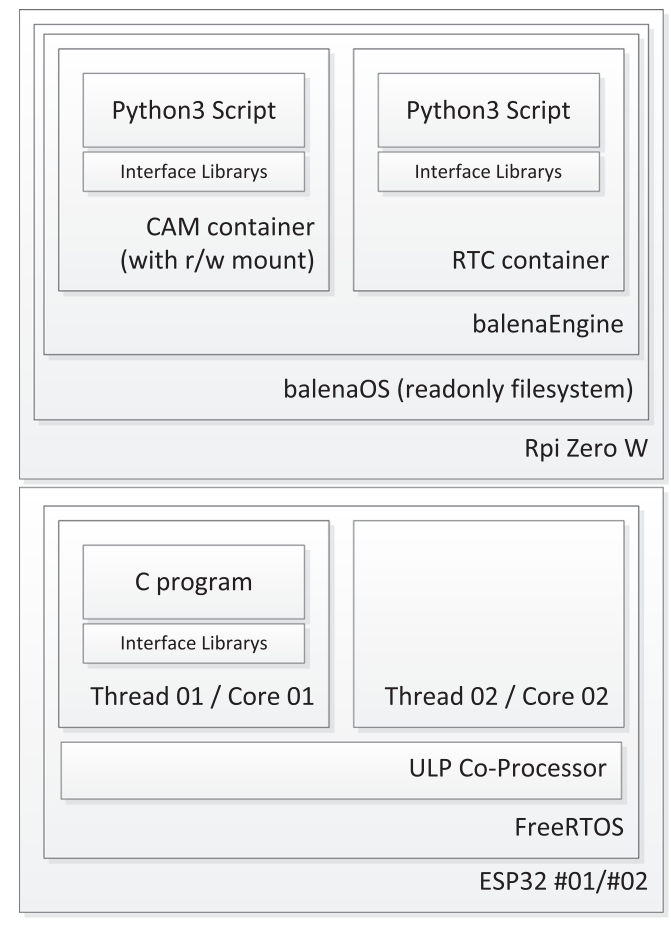

FIG. 3. apex software stack.

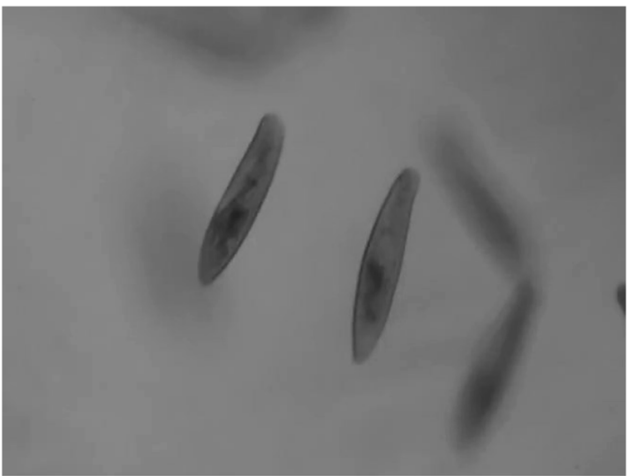

FIG. 4. Paramecium biaurelia ( $180 \mu \mathrm{m})$ in ROPUM, using the apex camera

it is already in use and the system will hold for a short randomized time and check again. If the logic level is high, it will pull the LR to a low level and announce thereby its usage of the i2c-bus to the other systems. With this simple design, all systems can coexist and use the sensors, while avoiding a bus stall due to concurrent access.

The Raspberry Pi Zero $\mathrm{W}$ has been used on apex to allow for a small, lightweight, low-power, and high performance Linux SBC. In the past, several iterations of the RPi have been flown on sounding rockets and used in CubeSat prototypes-particularly the full-sized "Model B" versions, which incorporate increasingly powerful processors-and additional peripherals-taking up more space, weight, and power than needed for most use cases. The smaller "Zero" model trades in these disadvantages for less processing power while still maintaining enough performance to work with high definition camera streams and additional signal processing.

This also sets it apart from the usage of systems like a GoPro: It takes up less space, weight, and is able to log signals and sensor data at the same time. The biggest problem with Linux SBCs is the reliance upon a perfect power supply. Especially, the sudden loss of

TABLE I. Comparison of ATMEGA328P and ESP32-PICO-D4.

\begin{tabular}{|c|c|}
\hline $\mathrm{MCU}$ & Features \\
\hline ATMEGA328P & $\begin{array}{l}1 \times 8 \text { bit, } 20 \mathrm{MHz} \text { CPU } \\
2 \mathrm{kB} \text { RAM } \\
32 \mathrm{kB} \text { flash } \\
23 \mathrm{GPIO}, 1 \times \text { UART, } 1 \times \mathrm{I} 2 \mathrm{C} \\
1 \times \text { SPI, } 6 \text { Ch. } 10 \text { bit ADC }\end{array}$ \\
\hline ESP32-PICO-D4 & $\begin{array}{l}2 \times 32 \text { bit, } 240 \mathrm{MHz} \text { CPU } \\
1 \times 32 \text { bit, ULP coprocessor } \\
520 \mathrm{kB} \text { RAM } \\
4 \mathrm{MB} \text { flash } \\
34 \mathrm{GPIO}, 3 \times \text { UART, } 2 \times \mathrm{I} 2 \mathrm{C}, 2 \times \mathrm{I} 2 \mathrm{~S} \\
4 \times \mathrm{SPI}, 18 \mathrm{Ch} .12 \text { bit SAR ADC } \\
\text { Secure boot, flash encryption, eFuse } \\
\text { Cryptoaccelerator }(\mathrm{AES}, \mathrm{SHA}-2, \mathrm{RSA}, \mathrm{ECC}) \\
\text { BT } 4.2 / \mathrm{BLE}, \mathrm{Wi}-\mathrm{Fi} 802.11 \mathrm{~b} / \mathrm{g} / \mathrm{n} / \mathrm{e} / \mathrm{i}\end{array}$ \\
\hline
\end{tabular}


power during a write cycle yields a considerable chance of corrupting the filesystem on the $\mu \mathrm{SD}$ card, making it unable to boot and perform its function. To circumvent this problem, the RPi on apex uses the former resinOS, now called balenaOS, a specialized read-only Linux OS. Due to its design, important files cannot be corrupted with an erroneous write process or power failure, which renders it to boot successfully in every case. The applications needed for the payload, in this case an implementation of the camera software, run in a virtualized container environment, thereby separated from other applications on the same system. This grade of separation is achieved using a balenaEngine, an implementation of the Moby (Docker) container engine for embedded devices. Even in case of one of the applications malfunctions, the other ones would not be affected. To finally solve the problem of the video recordings becoming corrupted after power-cycling during usage, the camera applications save real-time data in short bursts, which afterward can be recomputed to one continuous video stream for scientific analysis.

Due to the security requirements of the launch procedure, no active wireless systems are allowed on the rocket. As the ESP32 and RPi Zero W both include Wi-Fi and Bluetooth connectivity, these functionalities are hardwired switched off and are not initialized upon power-on.

As storage media, Samsung Evo Plus $\mu$ SD cards have been chosen, due to their predecessor usage on the ISS AstroPi program ${ }^{7}$ and their X-ray proof, waterproof, magnetism, and heat resistant design.

The overall construction of apex as a rapid developed prototype consists of a single $160 \mathrm{~mm} \times 100 \mathrm{~mm}$ eurocard/perfboard weighing $200 \mathrm{~g}$, excluding the experiment Rotation of Paramecium Under Microgravity (ROPUM).

To mount this eurocard onto its carrier, Cellfix II, a Fused Deposition Modeling (FDM) printed holder has been developed and used (Fig. 2).

\section{RESILIENCY DESIGN}

Due to the cost, limited availability, and repeatability associated with sounding rocket experiments, resiliency of an OBC is of utmost importance to safeguard against loss-of-science. Possible problems could arise due to damage induced from loss-of-power, power spikes, vibrations, heat during launch/re-entry, vacuum, and-with low probability - radiation. On apex, an isolated DC/DC converter with capacitors is used to protect the overall system against short loss-of-power and power spikes. It also protects the system against reverse polarity. All components on the board have been securely attached with a two-component epoxy and are held in place by using a Polylactic Acid (PLA) FDM printed holder, which is flexible enough to absorb bigger shocks, while staying rigid to counter up to $20.6 \mathrm{~g}$ encountered during descend. To counter the problem of overheating, system components have been selected for low energy consumption to achieve a high operational temperature range. This also meant to circumvent newer and more capable RPi models for the sake of lower power consumption and heat development. All components have been validated during two vacuum tests of $50 \mathrm{~min}$ and $72 \mathrm{~min}$ to ensure that even under these circumstances sensors and systems would stay operational without impact. The usage of radiation hardened $\mu \mathrm{SD}$ cards, which are also currently deployed on the ISS, is another step to make the system reliable from a hardware design perspective. However, to efficiently counter possible system malfunctions, software needs to be designed in a fault-tolerant way. The master-master configuration of the ESP32s in combination with the two radiation hardened $\mu \mathrm{SD}$ cards, as well as the two SPIFFS ring-memories, allows this subsystem to allow for the failure of one complete ESP32, both ring-memories, as well as one $\mu \mathrm{SD}$ card while still retaining full science with a slightly lower sampling frequency. The loss of both $\mu \mathrm{SD}$ cards and one ring-memory would still allow for the extraction of $1 \mathrm{~Hz}$ of scientific data. This kind of resiliency to environmental impacts is to be improved furthermore with apex Mk.II as described in the results of this paper.

As the camera cannot be easily attached to a second SBC, the design needs to be planned with overall system failure in mind. For a Linux computer, like the $\mathrm{RPi}$, the biggest concern is the power loss during write operation on its storage $\mu \mathrm{SD}$ card. The resulting data corruption would render the system unbootable, hence inoperable. The mitigation was to ensure that the OS is available on a read-only part of the $\mu \mathrm{SD}$ card, making data corruption outside of the scope of high radiation unlikely. Within this protected environment, the application software of the camera as well as the management system of the SBC has been separated via a container engine, protecting the camera from failures of the management. As the camera application does need writable storage to accommodate the video data, a fourth extended filesystem (ext4) partition has been mounted into the container. ext 4 as a journaling filesystem keeps track of changes to its data contents in contrast to traditional filesystems like second extended filesystem (ext2) or File Allocation Table 32 (FAT32) and therefore is more resilient against data loss. However, as this system is still prone to loss of data that are still opened/written, the only possibility to ensure that most of the data are secured is to record about 20 s of camera footage into a ring-buffer of the RPi's Random-Access Memory (RAM). From this buffer, $10 \mathrm{~s}$ pieces are written as single files into the mounted ext4 storage and synced to the filesystem. With this technique, the RPi is currently able to record a video with a maximum penalty of the loss of the last $60 \mathrm{~s}$ of the recorded video, even in the case of a complete power failure. This fact could even be improved with more aggressive tweaking of the settings. Due to this optimization, the default of complete data loss and an inoperable SBC after a power failure has been mitigated to an always working sensor with a maximum data loss of the last $60 \mathrm{~s}$ before power failure.

The apex goal does not only lie in presenting a more powerful OBC but also to ensure higher operational security and resilience in space operations (Fig. 3).

\section{EXPERIMENTS}

apex consists of multiple parts and the main purpose was to flight-approve each individual part and with that the overall system and compatibility with the MAPHEUS service module. For the MCUs, this means the function of the ESP32s, SPIFFS ringmemory, main-storage, link ready system, and i2c sensors. The SBC area should flight-proof the combination of the newly used RPi with balenaOS and RPi v2 camera. The $\mu$ SD card also contained $116 \mathrm{MB}$ of randomized test data of which the SHA256 checksum had been taken on ground before launch to see whether these data would be corrupted or changed during the brief flight. The camera of the RPi was used in this configuration to support the experiment ROPUM, which had the objective to monitor the behavioral change of the unicellular swimming organism Paramecium biaurelia 
under microgravity $(\mu \mathrm{g})$ using a microscope with FDM printed parts (Fig. 4).

\section{RESULTS}

ATEK/MAPHEUS-8 was launched on June 13, 2019 at 04:21:00 (CEST) from Esrange in Kiruna (Sweden). During the flight, $360 \mathrm{~s}$ of $\mu \mathrm{g}$ were achieved (roll rates $<1.0^{\circ} / \mathrm{s}$, altitude $>90 \mathrm{~km}$ ). The MCU subsystem started recording sensor data with power-on of the MAPHEUS service module at 02:53:34 (CEST) until the shutdown during descend at 04:34:20 (CEST). The SBC subsystem booted with power-on of the service module but remained idle until the Lift Off (LO) signal was sent. In addition, in this case, it was planned not to stop the recording by the Start of Data Storage (SoDS) signal, but with loss of power, to maximize collected data during the flight. The recording lasted for 12:15 min, which meant that the last minute of the flight was lost due to corruption of the writable memory space on the RPi. This was intended and showcased the correct implementation of the camera application. Would the file have been recorded in the traditional way, all footage of the flight would have been corrupted at this point, not only the last minute.

Regarding the results of both subsystems, the performance was as expected. The pair of ESP32, coupled with the LR system worked in a near perfect round-robin configuration, with the MCU\#01 recording $17.454 \mathrm{~KB}$ (64 203 data points) and the MCU\#02 17.452 KB (64 197 data points). The ring-memory of MCU\#01 contained $343 \mathrm{~KB}$ (1258 data points) vs MCU\#02 with $342 \mathrm{~KB}$ (1258 data points). The overall throughput was measured with 10.62 sensor captures/s and an MCU with little fluctuations between nine and 12 captures/s/MCU.

The $\mathrm{RPi}$, as reported, recorded 12:15 min of footage in black and white at 24 frames/s with a resolution of $1640 \times 1232$ pixels to facilitate the complete field of view of the implemented Sony IMX219 sensor. Additionally, it also logged the timestamps of its own boot-up, as well as all three signals [Lift Off (LO), Start of Experiment (SoE), Start of Data Storage (SoDS)]. It should be noted that with the exception of the hardwired LO signal, all other signals could be sent according to the users need. We used SoE and SoDS to mark the start and the end of the $\mu \mathrm{g}$ phase. With these data, it is now possible to analyze the changes in behavior of swimming cells under $\mu \mathrm{g}$, and the feasibility of ROPUM has been proven as well.

As for the sample payload of $116 \mathrm{MB}$ randomized test data, no corruption on the $\mu \mathrm{SD}$ card could be detected after recomputing the SHA256 checksums on earth.

\section{CONCLUSIONS AND PERSPECTIVES}

The new components used in apex worked reliably and accurately in the conditions of a sounding rocket experiment. While both MCUs worked with a sensor resolution of $10.62 \mathrm{~Hz}$, totaling at a little more than $21 \mathrm{~Hz}$, this is not yet the achievable peak performance. There is still potential for optimization, especially in the LR system, which sees headroom regarding its timing. In addition, reworking the prototype of apex from the eurocard prototype to a real Printed Circuit Board (PCB) will see better signal integrity for the i2c-bus and the possibility to use higher networking speeds while reducing the needed space significantly. Due to the positive results gathered with the test of this dual/master-master configuration, a short lab test of a three node, quorum-based system has been made.
In this setup, one node accesses the i2c-bus exclusively, which renders the time-intensive handover via the LR system unnecessary. The two remaining systems validate the operational status of the first node via a heartbeat protocol and can mutually agree to remove it from operations if it should fail. In this case, the two remaining nodes then fall back to the proven master-master configuration, using the LR system. Results of the lab test showcased that it is possible to achieve peak sensor sampling performance of up to $50 \mathrm{~Hz}$ while maintaining operational security and redundancy.

In the current configuration, only one of the two main cores of the ESP32s is used, while the coprocessor is also left on standby. For future missions, the test of the included cryptoacceleration for direct encryption of sensor values could be interesting, further enabling an encrypted and authenticated telecommand channel. As the telecommand interface on the ATEK/MAPHEUS- 8 mission was already overbooked, this could not be tested during this flight campaign.

As for the RPi/balenaOS camera, the current setup was tested in black and white/24 frames/s and a resolution of $1640 \times 1232$ pixels to retain the full field of view. Regarding the kind of application, needed resolution, and field of view, the used image sensor could record up to 90 frames/s at 720p resolution, however, with a partial field of view. The used RPi gives enough headroom to include future extensions, like a control system for different kinds of illuminations for the ROPUM microscope. In addition, the time between writing the real-time data to the $\mu \mathrm{SD}$ card could be optimized to allow for even more recoverable data after the flight.

For the future, apex could be developed into different kinds of modular applications: From a simple sensor carrier for university projects to a base station within the rocket communicating with deployable sensor platforms and picosatellites or a robust on-board computer for general purpose systems and experiment control.

\section{ACKNOWLEDGMENTS}

We want to thank the Institute of Material Physics in Space at the German Aerospace Center (DLR), Cologne, for vacuum testing as well as allowing us to fly on ATEK/MAPHEUS-8. We also acknowledge the support of the Mobile Rocket Base (MORABA) for flight certification of apex hardware as well as the on-site support with the Swedish Space Corporation (SSC) at Esrange during the campaign. This work was funded by the DLR Microgravity User Support Center (MUSC), Cologne.

\section{NOMENCLATURE}

\section{Acronyms}

apex advanced processors, encryption, and security

experiment
COTS commercial off-the-shelf

CSMA/CA carrier sense multiple access/collision avoidance

DLR German Aerospace Center

ext2 second extended filesystem

ext4 fourth extended filesystem 


$\begin{array}{ll}\text { FAT32 } & \text { file allocation table 32 } \\ \text { FDM } & \text { fused deposition modeling } \\ \text { i2c } & \text { inter integrated circuit } \\ \text { ISS } & \text { International Space Station } \\ \text { LO } & \text { lift off } \\ \text { LR } & \text { link ready } \\ \text { MCU } & \text { microcontroller unit } \\ \text { OBC } & \text { on-board computer } \\ \text { OS } & \text { operating system } \\ \text { PCB } & \text { printed circuit board } \\ \text { PLA } & \text { polylactic acid } \\ \text { RAM } & \text { random-access memory } \\ \text { ROPUM } & \text { rotation of paramecium under microgravity } \\ \text { RPi } & \text { Raspberry Pi } \\ \text { SBC } & \text { single board computer } \\ \text { SoDS } & \text { start of data storage } \\ \text { SoE } & \text { start of experiment } \\ \text { SPIFFS } & \text { SPI flash filesystem }\end{array}$

\section{REFERENCES}

${ }^{1}$ D. Geeroms, S. Bertho, M. De Roeve, R. Lempens, M. Ordies, and J. Prooth, "ARDUSAT, an Arduino-based cubesat providing students with the opportunity to create their own satellite experiment and collect real-world space data," in 22nd ESA Symposium on European Rocket and Balloon Programes and Related Research, 2015

${ }^{2}$ D. P. Violette, Arduino/Raspberry Pi: Hobbyist Hardware and Radiation Total Dose Degradation (NASA, 2014), pp. 1-15.

${ }^{3}$ W. Olsen, B. Wood, D. Rice, and J. R. Dennison, Microcontroller Survivability in Space Conditions (Utah State University, 2017).

${ }^{4}$ B. Peters, Ardusat Space Program: Training the Next Generation of Satellite Scientists and Engineers, SSC XIII-5 (Digital Commons, 2016).

${ }^{5}$ F. P. Kohn and J. Hauslage, "The gravity dependence of pharmacodynamics: The integration of lidocaine into membranes in microgravity," npj Microgravity 5, 5 (2019).

${ }^{6}$ IEEE, "Part 3: Carrier sense multiple access with collision detection (CSMA/CD) access method and physical layer specifications," Access 2008, 1-586.

${ }^{7}$ D. Honess, Astro Pi Upgrades on the International Space Station (Raspberry Pi Foundation, 2017). 